\title{
Working memory-driven attention improves spatial resolution: Support for perceptual enhancement
}

\author{
Yi Pan ${ }^{1} \cdot$ Qianying $\mathrm{Luo}^{2} \cdot$ Min Cheng ${ }^{3}$
}

Published online: 18 May 2016

(C) The Psychonomic Society, Inc. 2016

\begin{abstract}
Previous research has indicated that attention can be biased toward those stimuli matching the contents of working memory and thereby facilitates visual processing at the location of the memory-matching stimuli. However, whether this working memory-driven attentional modulation takes place on early perceptual processes remains unclear. Our present results showed that working memory-driven attention improved identification of a brief Landolt target presented alone in the visual field. Because the suprathreshold target appeared without any external noise added (i.e., no distractors or masks), the results suggest that working memory-driven attention enhances the target signal at early perceptual stages of visual processing. Furthermore, given that performance in the Landolt target identification task indexes spatial resolution, this attentional facilitation indicates that working memory-driven attention can boost early perceptual processing via enhancement of spatial resolution at the attended location.
\end{abstract}

Keywords Working memory $\cdot$ Attention $\cdot$ Perception . External noise

A number of studies have shown that the contents of working memory can bias attentional allocation toward memory-

Yi Pan

yipan@hznu.edu.cn

1 Department of Psychology, Hangzhou Normal University, Hangzhou, China

2 Zhejiang University of Finance and Economics, Hangzhou, China

3 Chengdu Technological University, Chengdu, China matching stimuli in the visual field, even when there is no explicit intention for observers to voluntarily shift attention to those stimuli (e.g., Calleja \& Rich, 2013; Carlisle \& Woodman, 2011; Downing, 2000; Han, 2015a; Huang \& Pashler, 2007; Olivers, Meijer, \& Theeuwes, 2006; Pan, 2010; Soto, Heinke, Humphreys, \& Blanco, 2005; Soto \& Humphreys, 2007). This memory-driven attentional capture cannot be attributable to the mechanism of bottom-up priming by stimulus repetition, as mere priming is usually not sufficient to guide attentional deployment (e.g., Olivers et al., 2006; Pan \& Soto, 2010; Soto et al., 2005) and distinct neural mechanisms have been found for attentional biases by working memory and repetition priming (Soto, Humphreys, \& Rotshtein, 2007; Soto, Llewelyn, \& Silvanto, 2012). Working memory contents are hence considered to play an important role in top-down attentional control by biasing attention in favor of the memory-matching item to resolve the competition for selection between multiple stimuli in the environment (Desimone \& Duncan, 1995), although there are some boundary constraints on the effects of working memory on visual attention (e.g., Dalvit \& Eimer, 2011; Downing \& Dodds, 2004; Han \& Kim, 2009; Kiyonaga, Egner, \& Soto, 2012; Kuo \& Chao, 2014; Pan \& Soto, 2010; Woodman \& Luck, 2007; Zhang, et al., 2011).

Previous research has shown consistent evidence that working memory-driven attention can improve manual response times (RTs) to visual targets when they appear at the location of the memory-matching stimuli (e.g., Downing, 2000; Soto, Heinke, Humphreys, \& Blanco, 2005). However, it remains unknown whether such an attentional effect takes place at the early perceptual stage of visual processing, as RTs may reflect non-perceptual rather than perceptual processes (Prinzmetal, McCool, \& Park, 2005). In order to determine the effect of working memory-driven attention on early perceptual processing, it is critical to measure 
accuracy or sensitivity to target identification in a perceptually challenging task as the primary dependent variable without pressing observers to respond quickly. To achieve this goal, Soto, Wriglesworth, Bahrami-Balani, and Humphreys (2010) included conditions where search displays were presented briefly and masked and observers were required to respond as accurately as possible within an unlimited time window. Relative to a neutral baseline without a match between working memory contents and search arrays, Soto et al. (2010) found improved identification sensitivity to search targets appearing at the location occupied by the memorymatching stimuli. Given that there was a null effect of bottom-up priming, they concluded that working memorydriven attention operates by enhancing early perceptual processing in a top-down manner at the location of the memorymatching stimuli.

It should be noted that because in the experiments of Soto et al. (2010) the target was always presented simultaneously with distractor stimuli in the visual field, there might have been uncertainty of the target location at the onset of search stimuli. Accordingly, the reason for reported improved identification of targets at the location of the memory-matching stimuli might be that working memory-driven attention operates post-perceptually to reduce spatial uncertainty of the target by prioritizing the memory-matching stimulus in visual search (Cosman \& Vecera, 2011). A recent study by Han (2015b) tried to address this issue by presenting observers with a response cue to reduce spatial uncertainty of the target in visual search. He found that identification accuracy for a target was improved when it appeared at the location of the memory-matching item despite that the target location was always indicated by a response cue at the onset of search stimuli. Thus, it has been demonstrated that working memory-driven attention can facilitate target identification without reducing target location uncertainty.

However, it still remains unknown so far whether the effects of working memory-driven attention arise directly through perceptual enhancement of target signal. We note that previous studies found the working-memory effects on identification performance only when the target was presented along with distractors and followed by masks (Cosman \& Vecera, 2011; Han, 2015b; Soto, et al., 2010). Because distractors and post-masks are thought to introduce visual noise that can interfere with target processing (Eckstein, 1998; Foley \& Schwartz, 1998), attention may improve identification accuracy or sensitivity to the attended target through diminishing the impact of the external noise introduced by distractors and/or masks (Dosher \& Lu, 2000a, 2000b; Shiu \& Pashler, 1994). Such attentional improvements in identification can be observed even without any change in the target signal. In this view, it is possible that the effects of working memory-driven attention on identification performance reported in previous research might not be attributed to enhancement of target signal, but rather to reduction of external noise.

The aim of the present study is to examine whether working memory-driven attention can directly improve the quality of the stimulus representation at the location of the memory-matching item. Previous research has shown that covert attention by spatial precueing enhances spatial resolution (i.e., our ability to discriminate small details in a visual stimulus) in the absence of external noise (e.g., Bocanegra \& Zeelenberg, 2011; Carrasco, Williams, \& Yeshurun, 2002; Golla, Ignashchenkova, Haarmeier, \& Their, 2004). This suggests that attentional effects on visual processing can be produced not through external-noise reduction and that attention can enhance perceptual representation of the stimulus at the attended location through finer spatial resolution. Motivated by this observation, here we investigated whether working memory-driven attention can enhance spatial resolution via signal enhancement of the target stimulus. Specifically, we asked whether working memory-based attention could improve spatial resolution at the attended location in the absence of external noise. Towards this end, we excluded added visual noise by presenting the target alone without any distractors or post-masks. Observers were asked to perform a spatial resolution task during the retention interval of a working memory task. Two taskirrelevant prime stimuli were simultaneously presented before the Landolt target designed specifically to probe spatial resolution. One of the prime stimuli matched the memory contents (memory-matching stimulus) and the other did not (mismatching stimulus). The target appeared at the location of either the memory-matching stimulus or the mismatching stimulus. It was predicted that working memory contents would guide attention in favor of the matching stimulus according to the biased completion model (Desimone \& Duncan, 1995). This top-down memory-driven attention may enhance the perceptual representation of the target through enhancement of spatial resolution at the attended location. On the other hand, attention is a very general term that refers to different selective processes. Given that working memory-driven attention does not influence certain aspects of visual processing such as temporal order judgment and motion perception (e.g., Pan \& Cheng, 2011; Turatto, Vescovi, \& Valsecchi, 2008), it might not enhance target signal via finer spatial resolution.

\section{Experiments 1 and 2}

\section{Method}

\section{Participants}

All observers reported having normal or corrected-to-normal visual acuity and normal color vision, and were naïve as to the purpose of the study. They were students at the Hangzhou 
Normal University, and participated for cash compensation. The number of participants was 20 for both Experiments 1 and 2 . There were different observers for the two experiments.

\section{Apparatus and stimuli}

The experiments were run on a Pentium IV computer with a processor speed of $2.4 \mathrm{GHz}$. The stimuli were displayed on a 17 -in. color monitor with a resolution of $1,024 \times 768$ pixels and a $75-\mathrm{Hz}$ refresh rate. Responses were made on a standard keyboard. The visual stimuli used in the memory task were geometrical shapes filled with different colors. The shapes could be a square $\left(2^{\circ} \times 2^{\circ}\right.$ of visual angle $)$, a circle $\left(2^{\circ} \times 2^{\circ}\right)$, a triangle $\left(2^{\circ} \times 1.7^{\circ}\right)$, or a diamond $\left(2^{\circ} \times 2^{\circ}\right)$. The color of the shapes could be red, green, blue, or yellow. The target in the spatial resolution task was a black Landolt $\mathrm{C}\left(1^{\circ} \times 1^{\circ}\right)$, with a $0.06^{\circ}$ gap on the left side or right side. All stimuli were presented on a gray background.

\section{Procedure and design}

Participants initiated each trial by pressing the space bar. A black central fixation cross $\left(0.3^{\circ} \times 0.3^{\circ}\right)$ was displayed for 1 , $000 \mathrm{~ms}$, followed by the memory cue presented at the center of the screen for $800 \mathrm{~ms}$. Participants were instructed to maintain their gaze at fixation without eye movements and memorize both the color and the shape of the memory cue to keep it in mind throughout the trial. After a delay of $507 \mathrm{~ms}$, two "prime stimuli" were simultaneously presented to the left and right of fixation for $200 \mathrm{~ms}$, separated by approximately $6^{\circ}$ of visual angle from center to center. Participants were told that the prime stimuli were task-irrelevant and they did not have to respond to them. After a 40-ms delay, a Landolt $\mathrm{C}$ with a gap on the left or the right was presented at the center of the location previously occupied by one of the two prime stimuli. The presentation duration of the Landolt $\mathrm{C}$ was $40 \mathrm{~ms}$ in Experiment 1 and was $27 \mathrm{~ms}$ in Experiment 2. Participants were instructed to indicate, as accurately as possible, which of two possible orientations of the Landolt $\mathrm{C}$ gap had been presented by pressing one of two buttons. Although observers were not pressed to respond quickly, response times were also recorded to evaluate the possibility of a speed-accuracy tradeoff. Immediately after a response to the Landolt target was made, an accuracy feedback sign was presented for $507 \mathrm{~ms}$. Then, a memory-test item was presented at the center of the screen and remained visible until response. Participants were required to indicate by a button-press whether or not the item matched the memory cue in both dimensions of color and shape (see Fig. 1).

The memory-test item and the memory cue had their color and shape in common on half of the total trials and they had just their color in common, just their shape in common, or neither attribute in common on the other half. The two prime stimuli were always different from each other in both dimensions of color and shape on each trial, with one exactly matching the memory cue (the memory-matching stimulus) and the other mismatching the cue (the mismatching stimulus). The Landolt target appeared at the location of the memory-matching stimulus on half of the trials (valid) and at the location of the mismatching stimulus on the other half (invalid). The locations of the memory-matching stimulus and the Landolt target as well as its gap-orientations were counterbalanced across trials. Participants were firstly familiarized with the tasks and performed 32 practice trials. Then, they performed four blocks of 64 trials each, for a total of 256 experimental trials.

\section{Results and discussion}

In Experiment 1, memory accuracy was $93.63 \%$ correct on average and did not differ significantly between valid and invalid trials, $F(1,19)=1.085, p=.311$, partial $\eta^{2}=.054$. To determine the effect of working memory-driven attention on spatial resolution, analyses of the Landolt target discrimination data included only trials on which memory responses were correct. As shown in Fig. 2, responses to Landolt targets were significantly more accurate on valid than on invalid trials, $F(1,19)=9.104, p=.007$, partial $\eta^{2}=.324$. The median RT results mirrored the accuracy data: participants responded faster on valid $(M=653 \mathrm{~ms})$ than on invalid trials $(M=672$ $\mathrm{ms}), F(1,19)=5.697, p=.028$, partial $\eta^{2}=.231$.

In Experiment 2, memory accuracy averaged $93.75 \%$ correct and did not vary between valid and invalid trials, $F<1$. As shown in Fig. 2, responses to Landolt targets were more accurate on valid than on invalid trials, $F(1,19)=9.435, p=$ .006 , partial $\eta^{2}=.332$. There was no sign of a speed-accuracy trade-off, since median RTs did not differ significantly between valid and invalid trials, $F<1$.

Taken together, the results from Experiments 1 and 2 showed that identification of a Landolt target was improved when the target appeared at the location previously occupied by the memory-matching item. This finding suggests that attention is biased to the location of the memory-match and increases spatial resolution at that location, given that performance in the Landolt target identification task indexes spatial resolution. Importantly, because the suprathreshold target was presented alone and hence there was no target location uncertainty at the onset of the target, our results cannot be accounted for by working memory-driven attention operating postperceptually to reduce spatial uncertainty of the target. Furthermore, given that the target was presented in the absence of added external noise (i.e., without distractors or masks), the improved identification accuracy could not be produced by the mechanism of external noise reduction. Thus, the pattern of results indicates that working memory- 


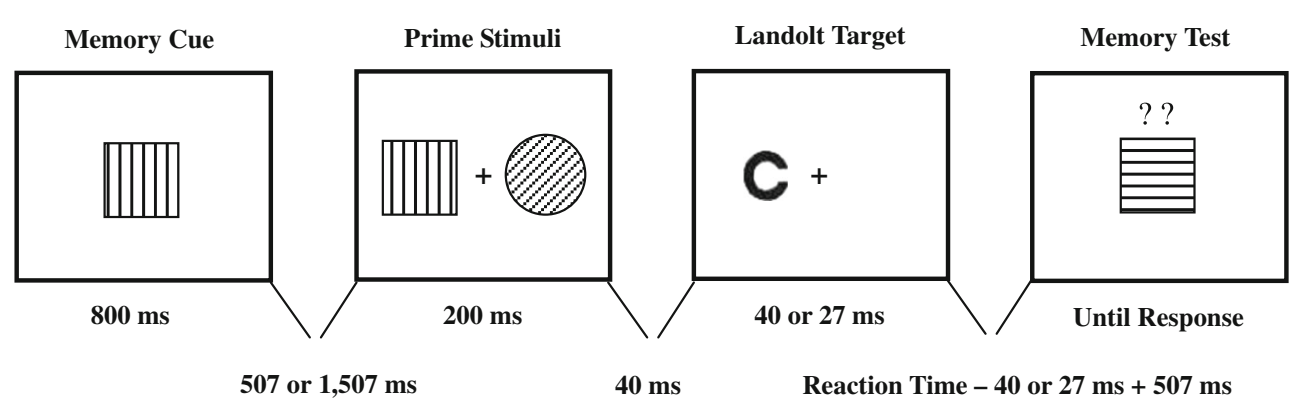

Fig. 1 Schematic illustration of the trial sequence and example stimuli in Experiments 1-5. The interstimulus interval between the memory cue and the prime stimuli was $507 \mathrm{~ms}$ in Experiments 1-4 and 1,507 ms in Experiment 5. The Landolt target was presented for $40 \mathrm{~ms}$ in

driven attention can enhance target signal through enhancement of spatial resolution.

\section{Experiment 3}

In this experiment, we further assessed whether the attentional improvements in spatial resolution were indeed due to working memory maintenance. Would such an attentional effect arise just as a result of the mechanism of repetition priming? Here we examined whether mere exposure to the object cue would produce the same effect.

\section{Method}

This was similar to that used in Experiment 2 with the following exceptions. A new group of 20 volunteers from the same pool participated. Participants were asked to attend to the object cue, but they received no explicit memory requirements and there was no memory test at the end of the trial. A go/no-

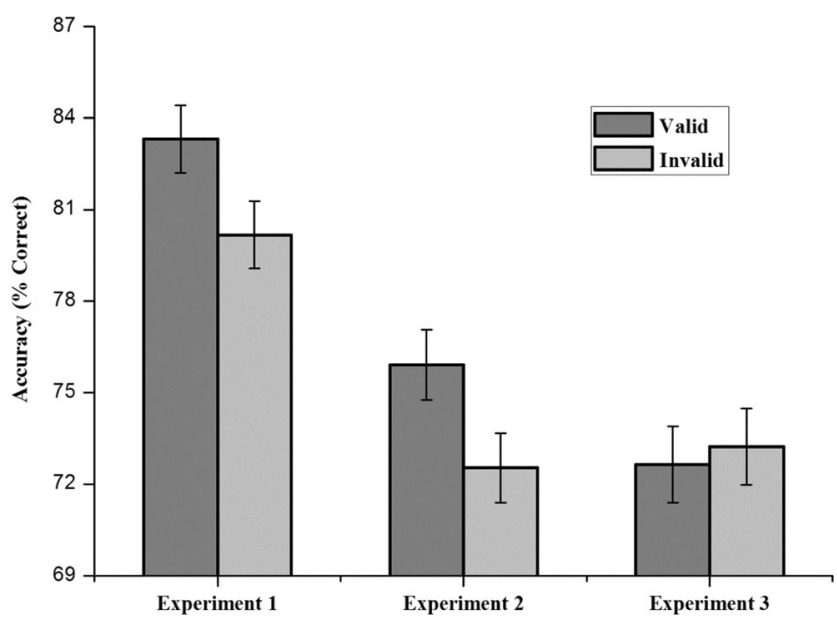

Fig. 2 Performance in the spatial resolution task for Experiments 1-3. Error bars represent within-subjects $95 \%$ confidence intervals, as described by Loftus and Masson (1994)
Experiment 1 and for $27 \mathrm{~ms}$ in Experiments 2-5. There was no memory test after the Landolt target in Experiment 3 or 5. Different patterns represent different colors

go procedure was used to enforce participants to process the cue. Observers were required not to carry out the Landolt target identification task when the cue was a red circle. Observers were told that the red circle would appear as a cue on $25 \%$ of total trials where they should end the trial by pressing the space bar. Participants were encouraged to perform the spatial resolution task as accurately as possible. Each participant performed 40 practice trials, followed by a total of 320 experimental trials.

\section{Results and discussion}

Observers performed appropriately as requested on catch trials where the cue was a red circle ( $95.23 \%$ correct on average). Analyses of the performance in the spatial resolution task showed that neither accuracy nor RTs varied significantly between valid and invalid trials, $F_{\mathrm{S}}<1$. The accuracy data are depicted in Fig. 2. This pattern of results suggests that merely priming an object's representation did not influence spatial resolution, although the object was perceptually identified. This is in line with the findings of previous studies that showed null effects of perceptual priming from a visual cue on visual search when the cue is irrelevant to the search task (e.g., Pan, Cheng, \& Luo, 2012; Soto, et al., 2005; 2010). We also compared accuracy on the spatial resolution task between Experiments 2 and 3, which yielded a significant interaction effect, $F(1,38)=6.112, p=.018$, partial $\eta^{2}=.139$. Thus, the attentional effects on spatial resolution observed in the first two experiments cannot be attributed to the mechanism of repetition priming.

\section{Experiment 4}

In the preceding experiments, attentional effects were computed based on the difference between valid and invalid trials. Here we sought to replicate the effect of working memorydriven attention on spatial resolution while including a neutral 
baseline condition where there was no matching between the memory cue and the prime stimuli. This allows us to specify the nature of the attentional effect mediated by working memory contents. In particular, we asked whether the effect is due to an increased resolution at the attended location, to a decreased resolution at the unattended location, or to both.

\section{Method}

This was similar to that of Experiment 2 with the following exceptions. One-third of the trials were valid and another third were invalid. The remaining third of the trials were neutral, and here neither of the two prime stimuli matched the memory cue. A new group of 20 volunteers participated in this experiment. Each participant performed 48 practice trials, followed by a total of 384 experimental trials. An adjustable chinrest helped participants to fixate the central cross throughout the trial. An eye-tracking system (Applied Science Laboratories, Model 504) was used to monitor eye movements and to ensure that fixation was maintained. Trials were excluded from the analyses if a saccade or a deviation from fixation exceeded $1^{\circ}$.

\section{Results and discussion}

Five percent of the trials, on average, were discarded due to saccadic eye movements or other significant deviations from central fixation. Memory accuracy averaged $95.62 \%$ correct and did not vary significantly across the three validity conditions, $F<1$. An analysis of accuracy data for the spatial resolution task showed that there was a significant main effect of validity, $F(2,38)=7.231, p=.002$, partial $\eta^{2}=.276$ (see Fig. 3). Pairwise comparisons indicated that responses to Landolt targets were significantly more accurate on valid trials relative to both invalid trials $(p=.004)$ and neutral trials $(p=$ $.025)$, which did not differ from each other $(p=.197)$. There

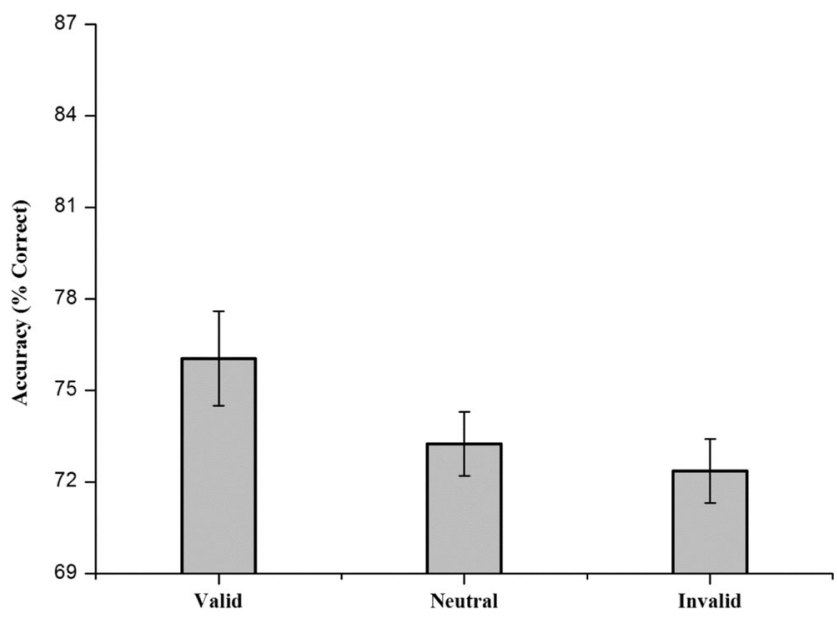

Fig. 3 Performance in the spatial resolution task for Experiment 4. Error bars represent within-subjects $95 \%$ confidence intervals, as described by Loftus and Masson (1994) was no sign of a speed-accuracy trade-off, because there was no significant effect of validity on median RTs, $F<1$. The results suggest that working memory-driven attention increases spatial resolution at the attended location without a decreased resolution at the unattended location. Thus, the attentional effect mediated by working memory contents reflects specifically the enhancement of spatial resolution at the attended location.

It could be argued that the attentional effects may be due to participants strategically attending to a memory-matching stimulus in order to refresh memory of the object cue and/or to facilitate discrimination of the target. This is because a memory test was always included on each trial and the target could appear at the location of the memory-matching stimulus, providing participants with a potential incentive for orienting toward the memory match when it was presented (cf. Kiyonaga, Egner, \& Soto, 2012). However, we believe the motivation to attend to a reappearing memory item, if it existed, was very weak, since memory performance was found not to benefit from the presence of the memory match relative to the neutral condition and the possibility of the target appearing at the location of the memory-matching stimulus was chance. Despite this, in Experiment 5 we sought to replicate the attention effects on spatial resolution, while further precluding any incentive to attend to the memory-matching stimulus.

\section{Experiment 5}

In this experiment, memory was only tested on surprise recognition trials where there were no prime stimuli and Landolt targets. Since memory was not probed after the onset of the prime stimuli, here there was no explicit incentive for attending to the memory-matching stimulus in order to refresh its representation. Moreover, to limit strategic orienting toward the memory-matching stimulus when the target was presented at its location, we reduced the proportion of the valid trials. Hence, the attentional effects in the present experiment, if any, would not be due to participants voluntarily attending to the memory item.

\section{Method}

This was similar to that of Experiment 4 with the following exceptions. A new group of 20 volunteers participated. Here, the prime stimuli were presented after $1,507 \mathrm{~ms}$ from the memory cue offset, and there was no memory test after the Landolt target identification task. In the experimental session there were 76 catch trials on which, instead of the prime stimuli, a memory probe was presented to ensure that participants actively maintained the object cue in working memory. There were a total of 384 critical trials on which there were $12.5 \%$, 
$37.5 \%$, and $50 \%$ of the trials for the valid, invalid, and neutral conditions, respectively.

\section{Results and discussion}

Accuracy for memory catch trials was high (96\% correct on average), indicating that participants were indeed keeping the cues in working memory as requested. On average, $6 \%$ of the critical trials were discarded from analyses of the performance in the spatial resolution task due to saccadic eye movements or other significant deviations from central fixation. An analysis of accuracy data for the spatial resolution task showed that there was a significant main effect of validity, $F(2,38)=$ $8.279, p=.001$, partial $\eta^{2}=.303$ (see Fig. 4). Responses to Landolt targets were significantly more accurate on valid trials relative to both invalid trials $(p=.003)$ and neutral trials $(p=$ $.008)$, which did not differ from each other $(p=.636)$. There was no sign of a speed-accuracy trade-off, since there was no significant effect of validity on median RTs, $F<1$. These results showed the attentional improvements in spatial resolution even when there was no explicit incentive for participants to attend to the memory-matching stimulus. Thus, the attentional effects by working memory on spatial resolution take place in an involuntary manner.

\section{General discussion}

Previous research has shown that working memory-driven attention can improve identification accuracy or sensitivity to a target when it appears at the location of the memorymatching item in the visual field (Han, 2015b; Pan, Cheng, \& Luo, 2012; Soto, et al., 2010). However, because the reported attentional effect occurred only under the condition where the target was always presented with distractors and followed

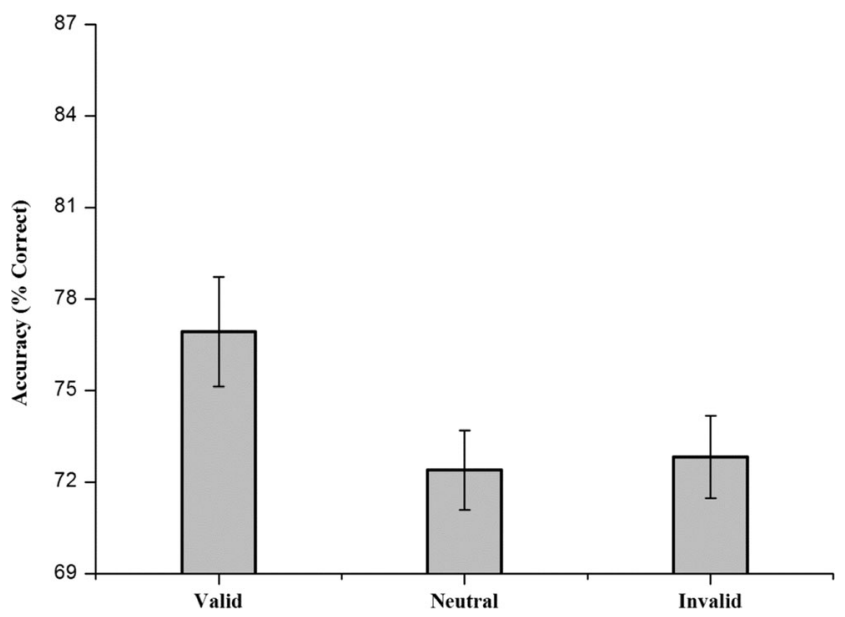

Fig. 4 Performance in the spatial resolution task for Experiment 5. Error bars represent within-subjects $95 \%$ confidence intervals, as described by Loftus and Masson (1994) by masks, it might be due to the mechanisms of spatial uncertainty reduction and/or external noise exclusion (Cosman \& Vecera, 2011; Dosher \& Lu, 2000a, 2000b; Shiu \& Pashler, 1994). The question then arises of whether working memorydriven attention can improve target identification through direct enhancement of target signal. By presenting the target alone without any external noise added (i.e., no distractors or postmasks), here we show that identification accuracy of a single target was improved when it appeared at the location previously occupied by the stimulus that matched the object cue held in working memory. This result is unlikely be attributable to the mechanism of repetition priming, because we failed to observe priming effects under the condition of mere exposure to the cue (see also Pan et al., 2012; Soto \& Humphreys, 2006; Soto et al., 2010). This suggests that the attentional facilitation is due specifically to working memory maintenance; attention is biased by working memory contents to the location of the memory-matching item and enhances the quality of the target representation at the attended location. Moreover, given that performance in the Landolt target identification task indexes spatial resolution, our results suggest that working memory-driven attention produces signal enhancement via finer spatial resolution of visual system.

The present work goes beyond previous studies, which established the effects of working memory-driven attention on perceptual identification of a target in the presence of external noise (Han, 2015b; Pan, Cheng, \& Luo, 2012; Soto \& Humphreys, 2006; Soto, et al., 2010). Here, we show that working memory-driven attention improves identification of a Landolt target with no external noise added, hence providing direct evidence suggesting that memory-based attention can enhance early perceptual processing through fine-grained spatial analysis at the attended location. Our results are consistent with a recent study by Hollingworth, Matsukura and Luck (2013). They found that rapid saccades to a single, abruptonset target were modulated by the contents of working memory, with saccades to memory-matching targets being generated more quickly and accurately than saccades to mismatching targets. This indicates that working memorybased overt attention can modulate perceptual strength of a stimulus in the absence of external noise. Because moving our eyes to a stimulus allows that stimulus to be processed within the fovea where spatial resolution is high, the results of Hollingworth et al. (2013) suggest that working memorydriven overt attention enhances perceptual representation of the memory-matching stimulus through finer spatial resolution. Given that eye movements were controlled in the present study, our findings further demonstrate that working memorydriven covert attention can intensify perception via enhancement of spatial resolution at the attended location in the visual periphery without moving the eyes.

The results may look somehow at odds with those of Cosman and Vecera (2011). They showed that the contents 
of working memory do not enhance sensitivity to a single masked target appearing at the location of the memorymatching stimulus. However, because the memory-matching item was presented alone in the absence of stimulus competition, the null effect may simply reflect a lack of memorydriven covert attention in their study. Indeed, according to the biased competition model, working memory contents guide covert attention especially when there are multiple stimuli competing for selection in the visual field (Desimone \& Duncan, 1995). Using a paradigm in which a memorymatching stimulus was presented simultaneously with a mismatching stimulus and followed by a single target, the present study creates a favorable opportunity for working memory to guide attention and provides direct evidence demonstrating enhancement of target signal by working memorydriven attention.

It has been proposed in the attention literature that signal enhancement and external noise reduction are two separate but not mutually exclusive mechanisms underlying attentional effects on visual processing (e.g., Carrasco, 2011; Carrasco, Williams, \& Yeshurun, 2002; Dosher \& Lu, 2000a; Lu \& Dosher, 2000). In this view, sensory enhancement may not be the sole mechanism for effects of working memorydriven attention on visual identification or discrimination. Reduction of external noise may also be an important source of attentional effects mediated by working memory contents. The mechanism of signal enhancement, by which memorydriven attention turns up the gain of the target signal, may play a prominent role in low-noise displays; the mechanism of noise reduction, by which memory-based attention diminishes the effects of external noise through filtering, may play a significant role in high-noise displays. Presumably, under certain conditions attentional facilitation by working memory may even be produced through a combination of signal enhancement and external noise reduction. Future research on this issue would be helpful to further specify the nature of the mechanisms underlying attentional effects mediated by working memory contents.

The importance of the present study is that it provides direct evidence for working memory-driven attention enhancing stimulus representation via finer spatial resolution. Our results suggest that top-down memory-based attention enhances spatial resolution just as does bottom-up spatial precueing of attention (e.g., Carrasco, Williams, \& Yeshurun, 2002). It is noteworthy, however, that these two forms of attention involve different neural networks (e.g., Soto, Mok, McRobbie, Quest, Waldman, \& Rotshtein, 2011) and do not influence all aspects of visual perception in a similar manner (e.g., Pan \& Cheng, 2011; Turatto, Vescovi, \& Valsecchi, 2008). Thus, attentional effects by working memory may not be directly inferred from those by spatial precueing and it is worth investigating them separately to specify the nature of their distinct underlying mechanisms.
In conclusion, the present study establishes a modest but reliable attentional improvement on spatial resolution mediated by working memory in a visual acuity task. The effect cannot be attributed to reduced external noise or target location uncertainty, as the suprathreshold target was presented alone without any distractors or masks added. Our results indicate that attentional modulation by working memory can take place at early stages of perceptual processing; memorydriven attention can improve the quality of the stimulus representation through enhancement of spatial resolution at the attended location.

Author note This research was supported by grants from the National Natural Science Foundation of China (grant number 31371034) and the Natural Science Foundation of Zhejiang Province (grant number LY13C090006).

\section{References}

Bocanegra, B. R., \& Zeelenberg, R. (2011). Emotional cues enhance the attentional effects on spatial and temporal resolution. Psychonomic Bulletin \& Review, 18, 1071-1076.

Calleja, M. O., \& Rich, A. N. (2013). Guidance of attention by information held in working memory. Attention, Perception, \& Psychophysics, 75, 687-699.

Carlisle, N. B., \& Woodman, G. F. (2011). Automatic and strategic effects in the guidance of attention by working memory representations. Acta Psychologica, 137, 217-225.

Carrasco, M. (2011). Visual attention: The past 25 years. Vision Research, 51, 1484-1525.

Carrasco, M., Williams, P. E., \& Yeshurun, Y. (2002). Covert attention increases spatial resolution with or without masks: Support for signal enhancement. Journal of Vision, 2, 467-479.

Cosman, J. D., \& Vecera, S. P. (2011). The contents of visual working memory reduce uncertainty during visual search. Attention, Perception, \& Psychophysics, 73, 996-1002.

Dalvit, S., \& Eimer, M. (2011). Memory-driven attentional capture is modulated by temporal task demands. Visual Cognition, 19, 145153.

Desimone, R., \& Duncan, J. (1995). Neural mechanisms of selective visual attention. Annual Review of Neuroscience, 18, 193-222.

Dosher, B. A., \& Lu, Z. L. (2000a). Noise exclusion in spatial attention. Psychological Science, 11, 139-146.

Dosher, B. A., \& Lu, Z. L. (2000b). Mechanisms of perceptual attention in precuing of location. Vision Research, 40, 1269-1292.

Downing, P. E. (2000). Interactions between visual working memory and selective attention. Psychological Science, 11, 467-473.

Downing, P. E., \& Dodds, C. M. (2004). Competition in visual working memory for control of search. Visual Cognition, 11, 689-703.

Eckstein, M. P. (1998). The lower efficiency for conjunctions is due to noise and not serial attentional processing. Psychological Science, 9, $111-118$

Foley, J. M., \& Schwartz, W. (1998). Spatial attention: Effect of position uncertainty and number of distracter patterns on the threshold-vs.contrast function for contrast discrimination. Journal of the Optical Society of America A, 15, 1036-1047.

Golla, H., Ignashchenkova, A., Haarmeier, T., \& Thier, P. (2004). Improvement of visual acuity by spatial cueing: A comparative study in human and non-human primates. Vision Research, 44, 1589-1600. 
Han, S. W. (2015a). Working memory contents revive the neglected, but suppress the inhibited. Cognition, 145, 116-121.

Han, S. W. (2015b). Working memory contents enhance perception under stimulus-driven competition. Memory \& Cognition, 43, 432-440.

Han, S. W., \& Kim, M.-S. (2009). Do the contents of working memory capture attention? Yes, but cognitive control matters. Journal of Experimental Psychology: Human Perception and Performance, $35,1292-1302$.

Hollingworth, A., Matsukura, M., \& Luck, S. J. (2013). Visual working memory modulates rapid eye movements to simple onset targets. Psychological Science, 24, 790-796.

Huang, L., \& Pashler, H. (2007). Working memory and the guidance of visual attention: Consonance-driven orienting. Psychonomic Bulletin \& Review, 14, 148-153.

Kiyonaga, A., Egner, T., \& Soto, D. (2012). Cognitive control over working memory biases of selection. Psychonomic Bulletin \& Review, 19, 639-646.

Kuo, C.-Y., \& Chao, H.-F. (2014). Role of attentional tags in working memory-driven attentional capture. Journal of Experimental Psychology: Human Perception and Performance, 40, 1301-1307.

Loftus, G. R., \& Masson, M. E. J. (1994). Using confidence intervals in within-subject designs. Psychonomic Bulletin \& Review, 1, 476490.

Lu, Z., \& Dosher, B. A. (2000). Spatial attention: Different mechanisms for central and peripheral temporal precues? Journal of Experimental Psychology: Human Perception and Performance, 26, 1534-1548.

Olivers, C. N. L., Meijer, F., \& Theeuwes, J. (2006). Feature-based memory-driven attentional capture: Visual working memory content affects visual attention. Journal of Experimental Psychology: Human Perception and Performance, 32, 1243-1265.

Pan, Y. (2010). Attentional capture by working memory contents. Canadian Journal of Experimental Psychology, 64, 124-128.

Pan, Y., \& Cheng, Q.-P. (2011). Do working memory-driven attention shifts speed up visual awareness? Attention, Perception, \& Psychophysics, 73, 2425-2433.

Pan, Y., \& Soto, D. (2010). The modulation of perceptual selection by working memory is dependent on the focus of spatial attention. Vision Research, 50, 1437-1444.

Pan, Y., Cheng, Q.-P., \& Luo, Q.-Y. (2012). Working memory can enhance unconscious visual perception. Psychonomic Bulletin \& Review, 19, 477-482.
Prinzmetal, W., McCool, C., \& Park, S. (2005). Attention: Reaction time and accuracy reveal different mechanisms. Journal of Experimental Psychology: General, 134, 73-92.

Shiu, L., \& Pashler, H. (1994). Negligible effect of spatial precuing in identification of single digits. Journal of Experimental Psychology: Human Perception and Performance, 20, 1037-1054.

Soto, D., \& Humphreys, G. W. (2006). Seeing the content of the mind: Enhanced awareness through working memory in patients with visual extinction. Proceedings of the National Academy of Sciences, 103, 4789-4792.

Soto, D., \& Humphreys, G. W. (2007). Automatic guidance of visual attention from verbal working memory. Journal of Experimental Psychology: Human Perception and Performance, 33, 730-737.

Soto, D., Heinke, D., Humphreys, G. W., \& Blanco, M. J. (2005). Early, involuntary top-down guidance of attention from working memory. Journal of Experimental Psychology: Human Perception and Performance, 31, 248-261.

Soto, D., Humphreys, G. W., \& Rotshtein, P. (2007). Dissociating the neural mechanisms of memory-based guidance of visual selection. Proceedings of the National Academy of Sciences, 104, 1718617191.

Soto, D., Wriglesworth, A., Bahrami-Balani, A., \& Humphreys, G. W. (2010). Working memory enhances visual perception: Evidence from single detection analysis. Journal of Experimental Psychology: Learning, Memory, and Cognition, 36, 441-456.

Soto, D., Mok, A. Y. F., McRobbie, D., Quest, R., Waldman, A., \& Rotshtein, P. (2011). Biasing visual selection: Functional neuroimaging of the interplay between spatial cueing and feature memory guidance. Neuropsychologia, 49, 1537-1543.

Soto, D., Llewelyn, D., \& Silvanto, J. (2012). Distinct causal mechanisms of attentional guidance by working memory and repetition priming in early visual cortex. Journal of Neuroscience, 32, 3447-3452.

Turatto, M., Vescovi, M., \& Valsecchi, M. (2008). On altering motion perception via working memory-based attention shifts. Journal of Vision, 8(5):11, 1-13.

Woodman, G. F., \& Luck, S. J. (2007). Do the contents of visual working memory automatically influence attentional selection during visual search? Journal of Experimental Psychology: Human Perception and Performance, 33, 363-377.

Zhang, B., Zhang, J. X., Huang, S., Kong, L., \& Wang, S. (2011). Effects of load on the guidance of visual attention from working memory. Vision Research, 51, 2356-2361. 